\title{
Bone Metastases of Endometrial Carcinoma: Report of Three Cases
}

\author{
(1) Nurefşan Aydın ${ }^{1}$, (1) Murat Danacıㄴ ${ }^{2}$, (1) ibrahim Yalçın ${ }^{3}$ \\ ${ }^{1}$ Ondokuz Mayıs University Faculty of Medicine, Medical Student, Samsun, Turkey \\ ${ }^{2}$ Ondokuz Mayıs University Faculty of Medicine, Department of Radiology, Samsun, Turkey \\ ${ }^{3}$ Ondokuz Mayıs University Faculty of Medicine, Department of Gynecological Oncology Surgery, Samsun, Turkey
}

\section{What is known on this subject?}

Endometrial cancer is the sixth most common cancer in women worldwide and the $14^{\text {th }}$ most common cancer overall. Endometrial cancer metastases to some parts of the body in a few ways. One of them is a hematogenous way. Bone metastasis occurs hematogenously and is a rare situation in literature. It has been reported 2-6\%. Generally, bone metastasis occurs in the pelvic bone and spine.

\begin{abstract}
What this study adds?
We presented three cases of bone metastasis in endometrial carcinoma. As we mentioned, bone metastases in endometrial carcinoma seldom occur. Due to this fact, these cases have significance for literature. These three cases' common point is bone metastases, while the process of each patient was different.
\end{abstract}

\section{ABSTRACT}

Presented herein are three patients with bone metastasis from endometrial cancer. Bone metastases were found in the pelvic bones of cases 1 and 3 and the spine of case 2 . All cases had an endometrial biopsy. Cases 1 and 2 were diagnosed as low-grade endometrioid carcinoma and case 3 as high-grade endometrioid carcinoma. Cases 1 and 2 had a total abdominal hysterectomy, bilateral salpingo-oophorectomy, and bilateral pelvic-paraaortic lymph node dissection. Case 2 received 6 cycles of chemotherapy after the operation and had a postoperative recurrence in the left paraaortic area 11 months after the surgery. This case report emphasized the significance of evaluation of the bone metastasis in endometrial cancer.

Keywords: Endometrial cancer, bone metastasis, pelvic bone, spine

\section{Introduction}

Endometrial cancer is the sixth most common cancer in women worldwide (1) with hematogenous metastases to some body parts (2). Bone metastasis hematogenously occurs and is rarely reported in the literature (2), with an incidence report of 2-6\% (3). Generally, bone metastasis occurs in the axial skeleton
(4). Herein, presented three cases with endometrial cancer with bone metastases and overviewed in the literature.

\section{Case Reports}

\section{Case 1}

A 60-year-old postmenopausal and multigravida female patient had magnetic

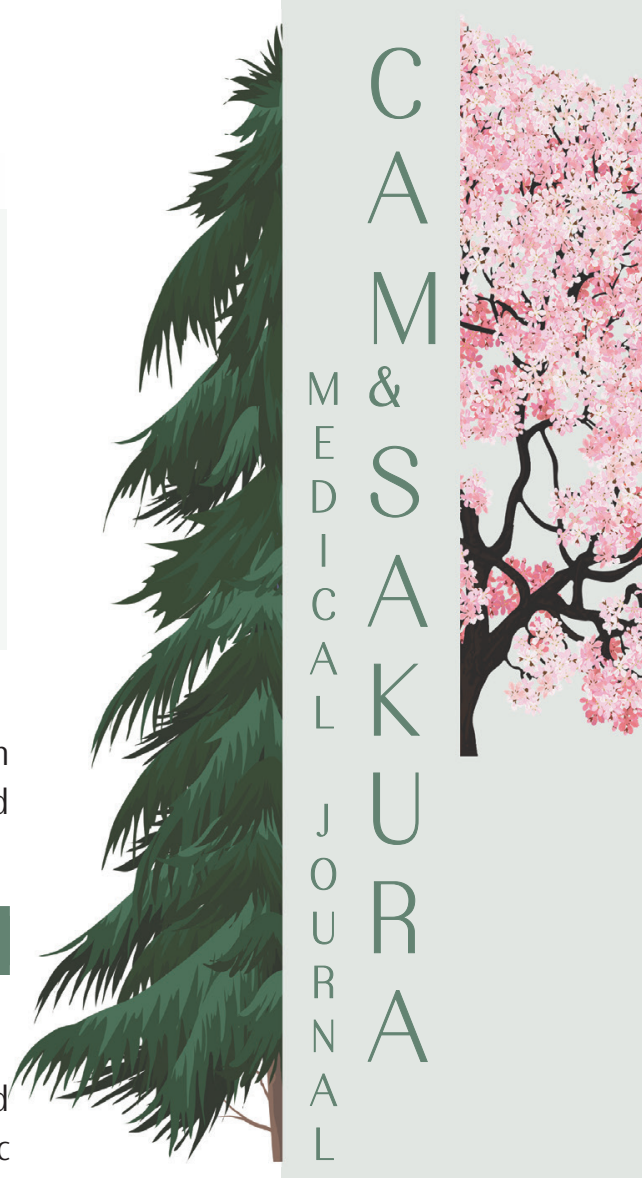

Address for Correspondence: Nurefşan Aydın Medical Student, Ondokuz Mayıs University Faculty of Medicine, Medical Student, Samsun, Turkey

Phone: +90 5394415799 E-mail: nurefsanaydin57@gmail.com 0RCID ID: orcid.org/0000-0002-6443-5738 Received: 21.09.2021 Accepted: 24.10.2021

(c) Copyright 2021 by the Cam \& Sakura Medical Journal published by Galenos Publishing House. 
resonance imaging (MRI), which pointed out a large uterus and a mass lesion that covered the whole uterine cavity walls and cervix and continues through the cervix. The mass did not cross the uterine borders. Pathological lymph nodes were found in the right posterior side of the external iliac vessels and the left medial side of the internal iliac vessel. A $13 \mathrm{~mm}$ diameter metastatic bone lesion was found at the pubic symphysis level (Figure 1). At the immunohistochemical staining, tumor cells were 90\% positive for estrogen and progesterone receptors. P53 was normal, which did not support the endometrial carcinoma with DNA mismatchrepair-mechanism defect. Ca125 increased at $67 \mathrm{U} / \mathrm{mL}$. The patient had a total abdominal hysterectomy, bilateral salpingo-oophorectomy, omental sampling, intra-abdominal cytology, bilateral pelvic-paraaortic lymph node dissection, sentinel lymph node sampling, removal of the mass above the bladder, and repair of serosa above the bladder. Pathology showed a grade 2 metastatic endometrioid carcinoma. The myometrial invasion was $75 \%$. The stromal involvement of the cervix was present. The lymphatic invasion was present, whereas the vascular was not. Peritoneal involvement above the bladder was observed. The peritoneal fluid was negative for malignancy. Additionally, 21 reactive lymph nodes were determined: 9 paraaortic lymph nodes, 1 left sentinel lymph node, 7 pelvic lymph nodes, 1 left pelvic lymph node, 1 left common lymph node, and 2 right common lymph nodes.

\section{Case 2}

A 63-year-old postmenopausal and multigravida female patient with MRI, showing a normal endometrium thickness according to age. Myomas were seen and the massive part of the tumor was located in the right adnexal and the right ovary, whereas the minor part was in the sinister and along the abdomen. Implants were seen in the upper abdomen and the larger one was in the Morrison pouch. No pathologic lymph nodes were observed. She had a debulking and bilateral pelvic-paraaortic lymph node dissection. The exploration revealed a $25 \mathrm{~cm}$ mass, which covered the whole abdomen and in the right ovary. Pathology showed that the tumor localization was the right and left ovary. Ovarian and endometrial cancers are not synchronous. Involvements include both ovarian surfaces, left uterine tube surface, and lymphovascular invasion. The omentum, the left uterine tube, and the left pelvic lymph nodes were involved. Peritoneal fluid was positive for malignancy. Additionally, 17 reactive lymph nodes and an implant in the left pelvis were observed. Endometrioid carcinoma grade 2 was diagnosed with chronic cervicitis, atrophic endometrium, and endometrial polyp. The patient received 6 cycles of chemotherapy for 4 months. After the chemotherapy, the last MRI revealed current pathologicalsized lymphatic nodes in the left paraaortic area. Ca-125 was 13.2 U/mL. Bone metastasis occurred in the vertebrae after cancer recurrence (Figure 2). The last Ca-125 was $26.86 \mathrm{U} / \mathrm{mL}$.

\section{Case 3}

A 60-year-old postmenopausal and multigravida woman applied to the hospital with a postmenopausal bleeding complaint. Endometrial biopsy indicated a malign tumor in the endometrium. Tumor cells were 90\% positive for estrogen and progesterone receptor, and p53 was normal, which did not verify the endometrial carcinoma with DNA mismatch-repairmechanism defect. The Papanicolaou test revealed cervicitis due to the presence of coccobacillus accordant with the flora change and malign atypical glandular cells. MRI showed a mass, which was full-layered and invaded the myometrium, reaching out the serosa and the cervix. The masses, which had equivalent characteristics with the uterine mass, were in the vagina, left ovary, and the right pubic bone (Figure 3). Ca125 was $120 \mathrm{U} / \mathrm{mL}$. Positron emission tomography (PET) indicated a hypermetabolic increased uterine wall thickness. Slightly increased involvement was observed in the lymph nodes of the bilateral internal iliac area, which was diagnosed

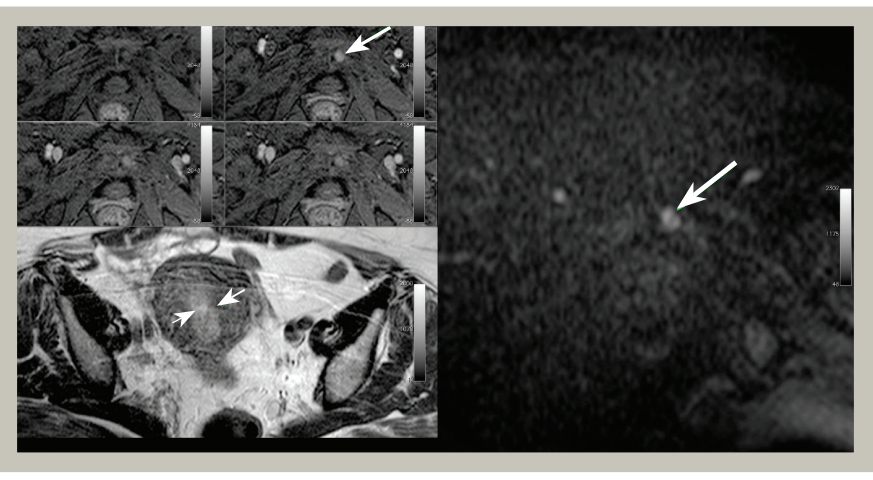

Figure 1. Long arrows indicate bone metastasis and short arrows indicate the endometrial carcinoma in the uterus

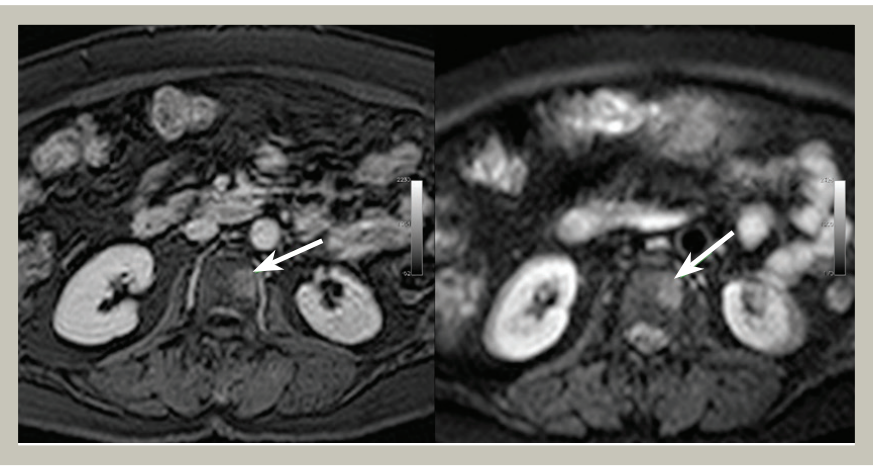

Figure 2. Vertebrae metastasis of a patient with recurrent endometrial cancer 


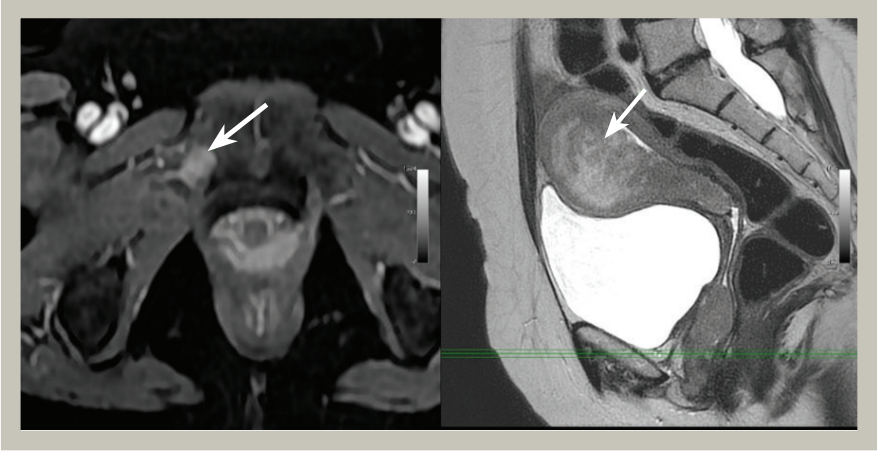

Figure 3. The left side of the picture shows bone metastasis in the right pubic bone. The right side of the picture shows endometrioid carcinoma in the uterus

as endometrioid carcinoma, grade 3. She also had a thyroid gland examination. Millimetric cystic areas and nodules with calcification were seen in the inferior pole of the right lobe. Except for the nodule, the parenchyma was homogeneous. No nodule was observed in the left lobe.

\section{Discussion}

This case report expressed bone metastasis in endometrial cancer. According to literature, bone metastasis is uncommon in endometrial cancer. Additionally, the spine and pelvic bones are mostly affected.

Despite the interaction between the increased Ca125 and lymph node involvement (5), case 2 had normal Ca125 values as reported. Bone metastasis, which is an extraperitoneal process, does not contribute to the Ca125 value because Ca125 rises as an intraperitoneal process consequence (5). Our data demonstrated that the Ca125 of cases with low-grade was lower than the case that has high-grade carcinoma.

The case with lymphovascular invasions can support that tumor cells enter the venous return directly and the systemic circulation indirectly (6). Cancer disseminates to the vertebrae and the pelvic bones by Batson's plexus and vertebral venous plexus $(6,7,8)$. Here, case 2 had lymphovascular invasion and bone metastasis in the vertebrae. This bone metastasis can be considered to occur by Batson's plexus and vertebral venous plexus.
Single bone metastasis is infrequent in endometrial carcinoma, thus second primary malignancy should be underlined (9). Hence, other different tests should be performed in addition to the routine process, e.g., mammography, thyroid gland ultrasound scan, etc. (9). Herein, case 3, who has thyroid nodules, had a thyroid gland examination. Consequently, bone metastasis was found to primarily originate from endometrial carcinoma.

Moreover, MRI and PET were used to detect bone metastases. Bone metastases could be detected by different scanning methods, such as computed tomography and bone scan (10). The bone scanning might be performed, but MRI and PET were more advantageous to detect other metastatic structures.

Females with bone metastases in endometrial cancer had higher age at diagnosis (11). Particularly, 95\% of cases are diagnosed after the age of 40 and in the postmenopausal process. The mean age of patients at diagnosis was 61 years, and they were all in the postmenopausal process.

Based on this report and other cases in the literature, bone metastasis should be considered in endometrial cancer diagnosis, especially symptoms of bone pain, to change the treatment methods and prognosis.

\section{Ethics}

Informed Consent: Patients have been informed before and after the research.

Peer-review: Externally peer-reviewed.

\section{Authorship Contributions}

Surgical and Medical Practices: M.D., I.Y., Concept: I.Y., Design: N.A., I.Y., Data Collection or Processing: N.A., Analysis or Interpretation: M.D., I.Y., Literature Search: N.A., Writing: N.A.

Conflict of Interest: No conflict of interest was declared by the authors.

Financial Disclosure: The authors declared that this study received no financial support. 


\section{REFERENCES}

1. Endometrial cancer. World Cancer Research Fund International (2021). Available from: https://www.wcrf.org/dietandcancer/ endometrial-cancer/Accessed September 192021.

2. Berek J, Hacker N. Berek et Hacker's gynecologic oncology. 6th ed. Philadelphia: Wolters Kluwer. 2015. p. 397.

3. Sahinler I, Erkal H, Akyazici E, Atkovar G, Okkan S. Endometrial carcinoma and an unusual presentation of bone metastasis: a case report. Gynecol Oncol 2001;82:216-218.

4. Özer H, Durmaz MS. Kemik Metastazları. Trd Sem 2021;9:137-155.

5. McEachron J, Chatterton C, Hastings V, et al. A clinicopathologic study of endometrial cancer metastatic to bone: Identification of microsatellite instability improves treatment strategies. Gynecol Oncol Rep 2020;32:100549.

6. Myriokefalitaki E, D'Costa D, Smith M, Ahmed AS. Primary bone metastasis as initial presentation of endometrial cancer (stage IVb). Arch Gynecol Obstet 2013;288:739-746.
7. Loizzi V, Cormio G, Cuccovillo A, Fattizzi N, Selvaggi L. Two cases of endometrial cancer diagnosis associated with bone metastasis. Gynecol Obstet Invest 2006;61:49-52.

8. Albareda J, Herrera M, Lopez Salva A, Garcia Donas J, Gonzalez R. Sacral metastasis in a patient with endometrial cancer: case report and review of the literature. Gynecol Oncol 2008;111:583-588.

9. Schöls WA, Kock HC, van Etten FH. Recurrent endometrial adenocarcinoma presenting as a solitary humeral metastasis. Gynecol Oncol 1995;59:148-150.

10. Kehoe SM, Zivanovic O, Ferguson SE, Barakat RR, Soslow RA. Clinicopathologic features of bone metastases and outcomes in patients with primary endometrial cancer. Gynecol Oncol 2010;117:229-233.

11. Ouldamer L, Bendifallah S, Body G, et al. Incidence, patterns and prognosis of first distant recurrence after surgically treated early stage endometrial cancer: Results from the multicentre FRANCOGYN study group. Eur J Surg Oncol 2019;45:672-678. 